\title{
ÖGUM-Mitteilungen
}

Österreichische Gesellschaft für Ultraschall

\section{in der Medizin}

\section{ÖGUM

\section{Erste ÖGUM-Studententutoren zertifiziert}

Nachdem das ÖGUM-interne studentische Ausbildungsprojekt zum zertifizierten „StudentInnentutor" mit Anfang des Jahres in die Praxis umgesetzt werden konnte, haben mittlerweile die ersten Teilnehmer eines Wahlfaches Abdomen (Kursleiter Stefan Meng) in Wien auch die ÖGUM-Zusatzqualifikation erfolgreich absolviert. Stefan Ritzal, Jacqueline Vetter, Aran Leitner, Jasmin Zessner-Spitzenberg und Georg Semmler dürfen wir als ersten zertifizierten „ÖGUMStudentInnentutoren“ recht herzlich zu der erfolgreichen Prüfung gratulieren!
Da auch in den anderen Bundesländern entsprechende Kurse im Laufen oder in Vorbereitung sind, werden wir weitere Zertifizierungen in diesem Jahr erwarten können und hoffen, durch diese Zusatzqualifikation schon früh in der medizinischen Ausbildung ein hohes Ultraschallausbildungsniveau etablieren zu können.

Wir freuen uns darauf, viele studentische Interssierte in Linz auf dem DLT begrüßen zu dürfen und laden zu einer regen Mitarbeit in der ÖGUM und in dem demnächst neu konstituierten „AK Studentische Ausbildung“ ein. Unsere ÖGUM-StudentenInnentutoren erhalten ein entsprechendes Zertifikat und werden als anerkannte StudentInnentutoren auf der Website aufgeführt, so dass sie als Ansprechpartner für KommilitonInnen und unsere Mitglieder in den jeweiligen Orten fungieren können, wenn qualifizierte studentische Tutoren gesucht werden. 